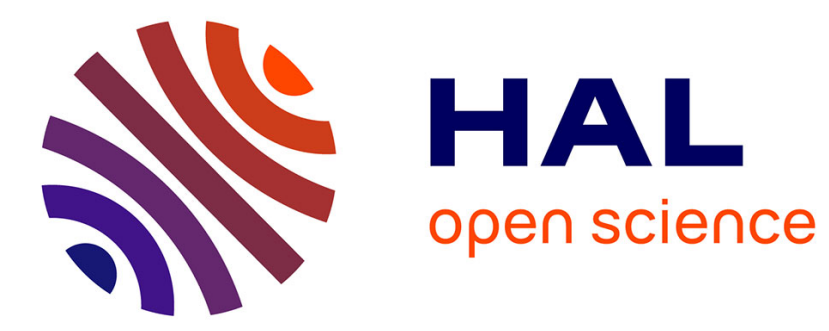

\title{
Linear dynamics of the Lamb-Chaplygin dipole in the two-dimensional limit
}

\author{
V. Brion, D. Sipp, L. Jacquin
}

\section{To cite this version:}

V. Brion, D. Sipp, L. Jacquin. Linear dynamics of the Lamb-Chaplygin dipole in the two-dimensional limit. Physics of Fluids, 2014, 26, pp.064103. 10.1063/1.4881375 . hal-01100934

\section{HAL Id: hal-01100934 \\ https://hal.science/hal-01100934}

Submitted on 7 Jan 2015

HAL is a multi-disciplinary open access archive for the deposit and dissemination of scientific research documents, whether they are published or not. The documents may come from teaching and research institutions in France or abroad, or from public or private research centers.
L'archive ouverte pluridisciplinaire HAL, est destinée au dépôt et à la diffusion de documents scientifiques de niveau recherche, publiés ou non, émanant des établissements d'enseignement et de recherche français ou étrangers, des laboratoires publics ou privés. 


\section{AIP $\left.\right|_{\text {Physics of }}$ \\ Fluids}

\section{Linear dynamics of the Lamb-Chaplygin dipole in the two-dimensional limit}

V. Brion, D. Sipp, and L. Jacquin

Citation: Physics of Fluids (1994-present) 26, 064103 (2014); doi: 10.1063/1.4881375

View online: http://dx.doi.org/10.1063/1.4881375

View Table of Contents: http://scitation.aip.org/content/aip/journal/pof2/26/6?ver=pdfcov

Published by the AIP Publishing

\section{Articles you may be interested in}

The laminar wake behind a $6: 1$ prolate spheroid at $45^{\circ}$ incidence angle

Phys. Fluids 26, 113602 (2014); 10.1063/1.4902015

On the lock-on of vortex shedding to oscillatory actuation around a circular cylinder

Phys. Fluids 25, 013601 (2013); 10.1063/1.4772977

Optimal transient disturbances behind a circular cylinder in a quasi-two-dimensional magnetohydrodynamic duct flow

Phys. Fluids 24, 024105 (2012); $10.1063 / 1.3686809$

Dipole evolution in rotating two-dimensional flow with bottom friction

Phys. Fluids 24, 026602 (2012); 10.1063/1.3680870

Two-dimensional vortex shedding of a circular cylinder

Phys. Fluids 13, 557 (2001); 10.1063/1.1338544

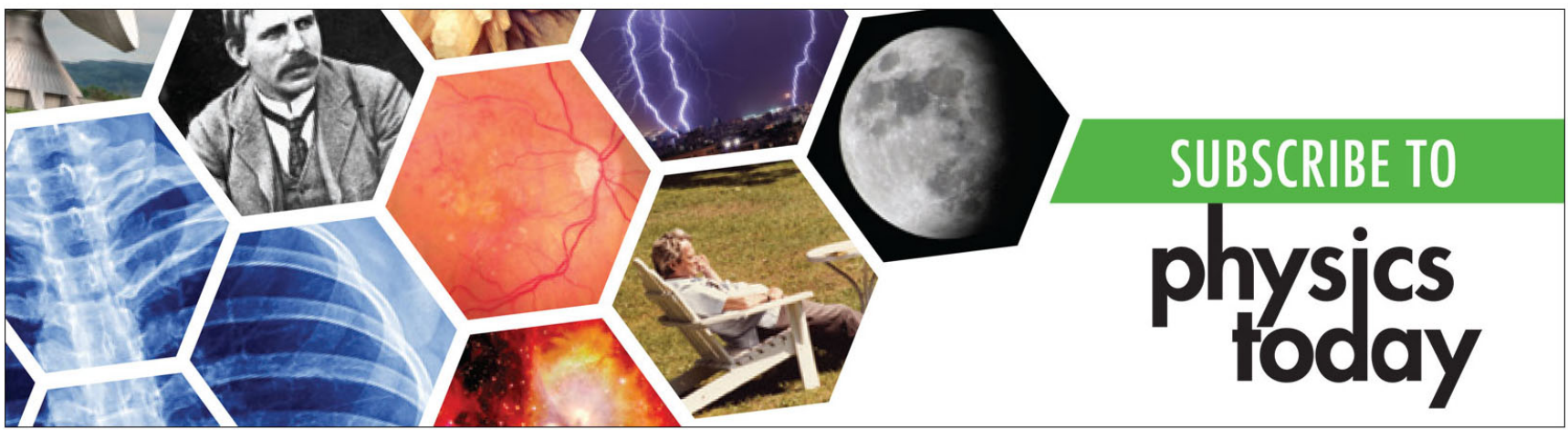




\title{
Linear dynamics of the Lamb-Chaplygin dipole in the two-dimensional limit
}

\author{
V. Brion, D. Sipp, and L. Jacquin \\ ONERA, 8 rue des Vertugadins, 92190 Meudon, France
}

(Received 26 June 2013; accepted 19 May 2014; published online 12 June 2014)

The dynamics of the Lamb-Chaplygin dipole in the large-wavelength limit is investigated by means of linear analysis. Taking the three-dimensional spectrum of the Lamb-Chaplygin dipole calculated by Billant ["Three-dimensional stability of a vortex pair," Phys. Fluids 11, 2069 (1999)] as a reference, we first show that additional families of unstable modes are present in the spectrum. Among them, a family of symmetric modes and one of antisymmetric modes, both present in the largewavelength limit, are the main topic of this investigation. The most unstable modes in these two families being purely two-dimensional, the two-dimensional dynamics is more particularly investigated. Our calculations show that the amplification rate of the antisymmetric instability is significantly larger than that of the symmetric instability. Also, while the two-dimensional symmetric mode is stationary and induces a shift of the dipole toward the upstream or downstream direction relatively to the dipole self-propagation velocity vector, the antisymmetric mode is unsteady and displaces the dipole into wavy oscillations about its initial straight trajectory. The leading physical mechanism of these two-dimensional instabilities is the vortex shedding that occurs downstream of the dipole. This shedding is the reaction of the external flow to the dipole motion and basically enables the conservation of the flow impulse. The destabilizing action of the wake is amplified as the displaced dipole generates more shedding. The dipole motion and the wake thus reinforce each other, leading to the instability. The strain mutually exerted by the vortices of the dipole, known as an essential mechanism of three-dimensional vortex pair instabilities, is also shown to participate to this destabilization. ( 2014 AIP Publishing LLC. [http://dx.doi.org/10.1063/1.4881375]

\section{INTRODUCTION}

This paper deals with the three-dimensional (3D) stability of the Lamb-Chaplygin dipole (hereafter referred to as the LCD) in the long-wavelength limit. The LCD flow has been described in detail by Meleshko and Van Heijst. ${ }^{1}$ While the 3D spectrum of the LCD has already been calculated by Billant et al., ${ }^{2}$ our calculations demonstrate the existence of additional modes. Some of them are in the long-wavelength limit where they dominate the three-dimensional dynamics in terms of amplification rate. In particular, two-dimensional (2D) modes are found that do not seem to have been described in the literature before, even when taking into account vortex pairs other than the LCD. The goal of the present paper is to present these 2D modes and to describe their dynamics in the linear regime.

It is important to note at first that there seems to be no clear understanding of the 2D stability of vortex pairs. In fact, the few available results appear conflicting. According to Saffman, ${ }^{3}$ counterrotating vortex patches should be stable to infinitesimal 2D disturbances but Saffman and Szeto ${ }^{4}$ found that pairs of counter-rotating vortices were unstable to antisymmetric disturbances and stable to symmetric ones. Yet the analysis of Saffman and Szeto ${ }^{4}$ is limited to the case of vortex patches and their results are mainly qualitative. Also the results of $\mathrm{Crow}^{5}$ obtained in the case of a pair of vortex filaments show that such a flow is only unstable in $3 \mathrm{D}$, and thus stable in $2 \mathrm{D}$. Finally, the 
results obtained by direct numerical simulations of 2D dipoles, such as those of Van Geffen and Van Heijst $^{6}$ for the Lamb-Chaplygin dipole or those of Delbende and Rossi ${ }^{7}$ for a generic dipole, show no sign of instability.

Conversely, the question of 2D stability is rather well described in the case of a single vortex. The flow made up of such a vortex embedded in a uniform and irrotational strain represents a simplified flow of a vortex pair, which is thus of direct interest to the present study. The 2D dynamics of a single vortex appears to be very rich. If we first consider the case where the strain is absent, theory predicts that the flow should be stable, however unsteady vortex solutions exist, such as the Kirchoff vortex which correspond to a steadily rotating ellipse that turns with angular velocity $\Omega=\omega_{0} a_{1} a_{2} /\left(a_{1}+a_{2}\right)^{2}$ where $a_{1,2}$ are the ellipse semi-axes and $\omega_{0}$ is the constant vorticity inside the patch. Within the limit of infinitesimal ellipticity $a_{1} \simeq a_{2}$, the Kirchoff vortices rotate at a rate of $\Omega=\omega_{0} / 4$.

The presence of strain makes the dynamics potentially unstable. Moore and Saffman ${ }^{8} \operatorname{showed}$ the existence of steady solutions to a vortex patch in the presence of strain, described by the relation

$$
\frac{\gamma}{\omega_{0}}=\frac{a_{1} a_{2}\left(a_{1}-a_{2}\right)}{\left(a_{1}+a_{2}\right)\left(a_{1}^{2}+a_{2}^{2}\right)}
$$

where $\gamma$ is the applied strain rate. The flow stability is then determined by the strain. For moderate strain $\left|\gamma / \omega_{0}\right|<0.15$ two solutions of the Moore and Saffman equation exist that differ in the value of $a_{1} / a_{2}$ relatively to a threshold value equal to 2.9 . The two solutions exhibit different linear stability behavior. The temporal exponent of both solutions is equal to

$$
\sigma_{m s}^{2}=\frac{\omega_{0}^{2}}{4}\left(\left(\frac{2 m a b}{a_{1}^{2}+a_{2}^{2}}-1\right)^{2}-\left(\frac{a_{1}-a_{2}}{a_{1}+a_{2}}\right)^{2 m}\right)
$$

which depends on the azimuthal periodicity $m$ of the disturbances (note that the subscript " $m s$ " refers to the names of the authors). This relation shows that the case $m=1$ is always unstable. The instability in this case corresponds to a translation of the ellipse in the outward direction of strain without any modification of the shape of the ellipse. This effect is well described by considering the stability of a point vortex in the presence of another point vortex of opposite circulation $\pm \Gamma$ (with $\Gamma>0$ ), the two vortices being separated by a distance $b$. It is straightforward to show that the position of one vortex moves in the direction of strain following

$$
\frac{d x}{d t}=\gamma y, \quad \frac{d y}{d t}=\gamma x
$$

in which the strain rate is

$$
\gamma=\frac{\Gamma}{2 \pi b^{2}}
$$

Robinson and Saffman ${ }^{9}$ also predicted this $m=1$ instability. Relation (1) also shows that unstable modifications of the ellipse shape occur for $m=2$ but only if $a_{1} / a_{2}>2.9$, the flow being otherwise stable.

The vortices of the LCD are characterized by $a_{1} / a_{2}=1.6$. The results of Moore and $\operatorname{Saffman}^{8}$ suggest that they should be unstable to the $m=1$ displacement instability. This will be shown to be one of the ingredients of the instabilities to which this work is dedicated, but not the entire story. As a remark, it is also worthwhile to note that while the $2 \mathrm{D}$ dynamics is free of the fundamental mechanisms of 3D instabilities (like the Crow and Widnall instabilities), such as bending, tilting, and stretching, the study of Moore and $\operatorname{Saffman}^{8}$ shows that the strain is still an active destabilizing mechanism.

In terms of applications, the question of the $2 \mathrm{D}$ stability of vortex pairs is of fundamental importance for all the situations in which the flow is constrained to be 2D. Geophysical flows, either in the atmosphere or in the ocean, belong to this category. The dynamics of cyclones and anticyclones, for instance, determines everyday weather. In aeronautics, contrails can be reasonably simplified as 2D flows and are important for determining the impact of aviation on climate change. In the ocean, dipoles can form by the roll-up of a jet flow out of a basin connected to a sea by a 
channel with tidal flushing (see Wells and Van Heijst ${ }^{10}$ ) and, for example, influence the deposition of sediments and the behavior of fish. Dipoles can also form when streams interact with coasts, for instance, around the southern coast of Madagascar (see De Ruijter et al. ${ }^{11}$ ) or in the wake of islands. Khvoles $\mathrm{et} \mathrm{al.}{ }^{12}$ investigated the stability of modons with respect to various forms of perturbation in the context of geophysical flows. Even if the results do not apply to the present case because the Coriolis force is not taken into account here, the oscillations of the modon about its centerline and the emission of vorticity observed by Khvoles ${ }^{12}$ bear a close resemblance to the dynamics of the antisymmetric mode detailed in the present paper. In the context of $2 \mathrm{D}$ turbulence, Dritschel ${ }^{13}$ investigated the interactions of vortex pairs. He showed how an initial well-balanced dipole exhibits an antisymmetric destabilization that provokes the removal of vorticity from one of the vortices and a subsequent return of the pair to an equilibrium state. This short review shows that a better understanding of the dynamics of vortex pairs is of high interest to progress with the interpretation of several practical problem in fluid mechanics.

Our approach in this paper is first to recalculate the three-dimensional spectrum of the LCD that was found by Billant $e t a .^{2}$ and then to point out the modes in the large-wavelength limit that had not been described in this previous work. Two families of modes are found, one belonging to the subset of symmetric modes $\hat{\boldsymbol{u}}_{S}$ and the other belonging to the antisymmetric subset $\hat{\boldsymbol{u}}_{A}$. Hereafter, we will use the capital letters $A$ and $S$ to denote the antisymmetric and symmetric subsets, respectively, and we will largely make reference to mode $A$ and mode $S$ in the core of the article. It will be shown that the mode $\mathrm{S}$ corresponds to a growing shift of the dipole position either upstream or downstream and that the mode A induces growing oscillations of the dipole about its natural, undisturbed trajectory.

The paper is organized as follows. In Sec. II, we present the method for the linear stability analysis and the numerical framework. In Sec. III, we first present the complete spectrum of the unstable modes associated with the $\mathrm{S}$ and A subsets. Most of the modes are those that have already been found by Billant $e t$ al. ${ }^{2}$ However, we highlight one family of modes in the A subset and another one in the S subset. We analyse the associated linear dynamics in Secs. IV A-IV C and propose a physical scenario to explain how these instabilities develop in Sec. V.

\section{STABILITY ANALYSIS}

\section{A. Governing equation}

We consider the flow made up of two vortices whose evolution is described by the Navier-Stokes equations in their incompressible form

$$
\begin{aligned}
& \nabla \cdot \boldsymbol{u}=0, \\
& \frac{d \boldsymbol{u}}{d t}=-\nabla p+v \Delta \boldsymbol{u} .
\end{aligned}
$$

Cartesian coordinates $\boldsymbol{x}=(x, y, z)$ are used throughout this study. The vortex axis is $z$, and $x$ and $y$ are the space coordinates in the transverse directions. In this paper, $x$ is along the horizontal and $y$ is along the vertical. The vorticity vector is noted $\omega=(\xi, \eta, \zeta)$ (note that we use the scalar $\omega$ later on to denote the frequency). We suppose that the vortices are slightly perturbed and therefore decompose the flow as a sum comprising a base flow, namely, the stationary and 2D vortex pair, and three-dimensional unsteady perturbations. The velocity field associated with the perturbations is $\boldsymbol{u}^{\prime}=(u, v, w)$ and that of the base flow is $\overline{\boldsymbol{u}}=(\bar{u}, \bar{v}, 0)$. Note that the base flow depends only on $x$ and $y$ and is assumed parallel in the $z$ direction. The pressures are noted $p^{\prime}$ and $\bar{p}$. The total velocity field writes $\boldsymbol{u}=\overline{\boldsymbol{u}}+\epsilon \boldsymbol{u}^{\prime}$ with $\epsilon$ taken infinitesimal (same for pressure). The equation for the perturbation dynamics is obtained by introducing the velocity field decomposition into the Navier Stokes equations (4) and neglecting all $O\left(\epsilon^{2}\right)$ terms

$$
\begin{aligned}
\nabla \cdot \boldsymbol{u}^{\prime} & =0, \\
\frac{\partial \boldsymbol{u}^{\prime}}{\partial t}+\overline{\boldsymbol{u}} \cdot \nabla \boldsymbol{u}^{\prime} & =-\boldsymbol{u}^{\prime} \cdot \nabla \overline{\boldsymbol{u}}-\nabla p^{\prime}+v \Delta \boldsymbol{u}^{\prime} .
\end{aligned}
$$


Let us introduce $\mathrm{O}$ the point located midway between the two vortices. Due to the symmetry of the base flow about the $(\mathrm{Oxz})$ plane, perturbations can be divided into two subsets. The first subset comprises A perturbations $\boldsymbol{u}_{A}^{\prime}$ which are characterized by even axial vorticity about the (Oxz) plane. The second subset comprises S perturbations $\boldsymbol{u}_{S}^{\prime}$ which correspond to perturbations with odd axial vorticity about the $(\mathrm{Oxz})$ plane. This writes

$$
\boldsymbol{u}_{A}^{\prime}: \zeta^{\prime}(x,-y, z)=\zeta^{\prime}(x, y, z), \quad \boldsymbol{u}_{S}^{\prime}: \zeta^{\prime}(x,-y, z)=-\zeta^{\prime}(x, y, z) .
$$

A convenient manner to interpret these symmetries is the following: $S$ perturbations lead to symmetric deformations of the vortices about the symmetry plane while A perturbations lead to deformations with a twofold symmetry with respect to the mid point $\mathrm{O}$ between the two vortices of the pair.

Note that the axial component of the $\mathrm{S}$ and A perturbation vorticity can be obtained through the following relations:

$$
\zeta_{A}^{\prime}(x, y, z)=\frac{\zeta^{\prime}(x, y, z)+\zeta^{\prime}(x,-y, z)}{2}, \quad \zeta_{S}^{\prime}(x, y, z)=\frac{\zeta^{\prime}(x, y, z)-\zeta^{\prime}(x,-y, z)}{2} .
$$

The base flow being $2 \mathrm{D}$, the perturbations can be additionally decomposed into axial waves of complex amplitude $\hat{\boldsymbol{u}}(x, y, t)=(\hat{u}, \hat{v}, i \hat{w})$ (note the absence of dependence on $z$ here), i.e.,

$$
\boldsymbol{u}^{\prime}(x, y, z, t)=\hat{\boldsymbol{u}}(x, y, t) e^{i k z}+c . c .
$$

where c.c. stands for the complex conjugate and $k$ is the axial wavenumber, which is real in the present case as we perform a temporal stability analysis.

\section{B. Base flow}

The LCD is chosen as the base flow. The LCD is the juxtaposition of a rotational flow contained inside a disc of radius $D / 2$ and an irrotational flow that goes past the disc at an incoming speed $U$. The LCD is a steady solution of the steady Euler equations. It can be characterized by the relationship between its vorticity field $\bar{\zeta}$ and its stream function $\bar{\psi}$. Inside the disc, the relationship is linear. Meleshko and Van Heijst, ${ }^{1}$ who provide the theoretical background for the LCD flow, use a constant $\beta$ such that $\bar{\zeta}=-\beta^{2} \bar{\psi}$. Outside the disc, $\beta$ is nil. Note that it is fair to mention that several authors mentioned the LCD flow prior to Meleshko and Van Heijst ${ }^{1}$ but this is clearly stated in their article so the interested reader can learn the complete history there.

This linear relationship is a particular solution of the equation $\Delta \bar{\psi}=f(\bar{\psi})$ characterizing steady solutions of the Euler equations (note that other solutions of this kind are given by Hesthaven et $a l .{ }^{14}$ ). Introducing $r$ and $\theta$ the polar coordinates in the (Oxy) plane, the stream function of the LCD is given by

$$
\bar{\psi}(r, \theta)=\left\{\begin{array}{ll}
C J_{1}(\beta r) \sin \theta & \text { if } r \leq D / 2 \\
U r\left(1-\frac{D^{2}}{4 r^{2}}\right) \sin \theta & \text { if } r \geq D / 2
\end{array},\right.
$$

where $J_{1}$ is the Bessel function of the first kind and

$$
C=-\frac{2 U}{\beta J_{0}(\beta D / 2)}
$$

The velocity $U$ can also be interpreted as the propagating velocity of the dipole. Indeed, the dipole is self-propelled due to the induction of each vortex upon the other. The value of $\beta$ is imposed by the continuity of the tangential velocity at the boundary of the dipole and is thus chosen such that $J_{1}(\beta D / 2)=0$. This condition yields $\beta D=2.4394 \pi$ and finally gives $U=0.91982 \Gamma /(\pi D)$ where again, $\Gamma$ is the circulation of one vortex.

Figure 1 shows the streamlines and the base flow axial vorticity $\bar{\zeta}$ in the reference frame attached to the vortex pair. The separating streamline between the rotational and the external flow is usually denoted as the Kelvin oval, and is a circle of radius $R=D / 2$ in the present case. The flow outside the oval goes from left to right as indicated by the black arrows. All quantities are referenced on the propagating velocity $U$ and dipole diameter $D$. Thereafter we will use the superscript “*” to denote 


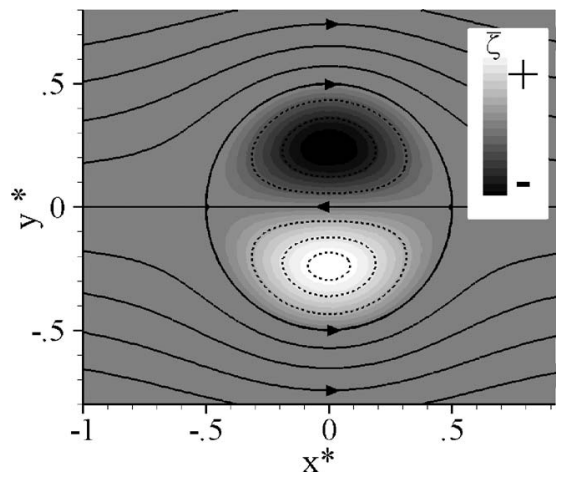

FIG. 1. Contours of the axial vorticity $\bar{\zeta}$ and streamlines of the LCD.

normalized quantities, such as the non-dimensional wavenumber $k^{*}=k D$. The Reynolds number writes $R e=U D / \nu$.

An important parameter for the vortex dynamics is the aspect ratio $a / b$ of the dipole, where $a$ is the dispersion radius defined by

$$
a^{2}=\frac{\int_{y>0}\left[\left(x-x_{c}\right)^{2}+\left(y-y_{c}\right)^{2}\right]|\bar{\zeta}| d x d y}{\Gamma}
$$

with $\left(x_{c}, y_{c}\right)$ taken as the vorticity centroid of the top vortex. The values of $a, b$, and $a / b$ can be useful to draw comparisons so we give them here, based on the diameter $D: b \simeq 0.46 D, a \simeq 0.206 D$, and $a / b \simeq 0.45$.

In the linear stability analysis, we suppose that the base flow is frozen. While this is not rigorously the case since the baseflow diffuses due to viscosity, this approach is classic in vortex stability analysis. The reasoning behind it is that the perturbation time scale $t_{\text {perturbation }}$ is much smaller than the baseflow time scale $t_{\text {base }}$ associated with viscous diffusion. However, in the light of the results obtained in the present work, the question of the frozen baseflow hypothesis appears more difficult than for the classic approach, because viscous diffusion at the perturbation order plays an important role in the instability dynamics. A thorough analysis of the problem is only achieved in Sec. VI at the end of the article, once all the information derived from this work is available, and concludes the validity of this approach.

As for now, we only recall the case of 3D perturbations which is the usual basis for this hypothesis. The perturbation time scale is that associated with the mutual strain rate $\gamma$ between the vortices, already given in (3). As a consequence, $t_{\text {perturbation }} \simeq \gamma^{-1}=2 \pi b^{2} / \Gamma$. The time scale of the base flow is associated with the viscous diffusion of the vortices and writes $t_{\text {base }} \simeq a^{2} / \nu$. The ratio $r_{t}=t_{\text {base }} / t_{\text {perturbation }}$ is what matters to justify the frozen base flow approach. This ratio is equal to $r_{t}=\Gamma /(2 \pi \nu)(a / b)^{2}$ and simple algebra leads to $r_{t}=0.5 \operatorname{Re}(a / b)^{2}$ if one uses the approximation $\Gamma \simeq \pi D U$. This last expression is based on the point vortex approximation of the drift velocity of the vortex pair $U \simeq \Gamma /(2 \pi b)$. Moreover, we use $b \simeq D / 2$ according to the values provided in the previous paragraph. Note that the true drift velocity is in fact lower than this point vortex model (see Delbende and $\operatorname{Rossi}^{7}$ for more details) but we neglect this effect here. The frozen base flow is justified if the Reynolds number is large enough to compensate for the small value of $(a / b)^{2}$.

Another point of concern is the vorticity profile of the LCD, which is not differentiable at the oval boundary as a result of the absence of viscosity in the Lamb-Chaplygin model. This peculiarity has never been mentioned by any previous authors but could play a role on the perturbation dynamics according to the linearized equation for the axial vorticity in which the derivatives of the base flow vorticity appear. It turns out that the sharpness of the vorticity profile at the oval boundary has no effect on the stability results. Indeed, we considered the stability of a smooth dipole that has no such sharpness in its vorticity profile. By performing the same stability analysis (which is detailed in Appendix A) we found the same 2D unstable modes. This smooth dipole was obtained by a 
numerical simulation of an initial flow composed of two Lamb-Oseen vortices as in Sipp et al. ${ }^{15}$ and is characterized by an aspect ratio $a / b=0.3$ close to that of the LCD.

\section{Global modes and numerics}

The temporal stability of the base flow is investigated by looking for positive values of the amplification rate of the global modes $(\tilde{\boldsymbol{u}}, \tilde{p})$ defined by

$$
(\hat{\boldsymbol{u}}, \hat{p})=(\tilde{\boldsymbol{u}}, \tilde{p})(x, y) e^{i k z+(\sigma+i \omega) t},
$$

where $\sigma$ is the growth rate and $\omega$ is the frequency of the global mode. The velocity and pressure fields are defined spatially by $\tilde{\boldsymbol{q}}(x, y)=(\tilde{u}, \tilde{v}, \tilde{w}, \tilde{p})$. The introduction of the global mode decomposition into the linearized equations (5) leads to the following eigenvalue problem which is parameterized by $k$ and $R e$ :

$$
A_{k, R e} \tilde{\boldsymbol{q}}=(\sigma+i \omega) B \tilde{\boldsymbol{q}},
$$

where matrices $A_{k, R e}$ and $B$ are given by

$$
A_{k, R e}=\left(\begin{array}{cc}
{\left[-\overline{\boldsymbol{u}} . \nabla+\frac{1}{R e} \Delta\right] \mathcal{I}-\nabla \overline{\boldsymbol{u}}} & -\nabla \\
\nabla^{T} & 0
\end{array}\right) B=\left(\begin{array}{cc}
\mathcal{I} & 0 \\
0 & 0
\end{array}\right),
$$

where ${ }^{T}$ stands for the conjugate transpose operator, $\mathcal{I}$ is the identity matrix, $\Delta=\partial_{x x}+\partial_{y y}-k^{2}$, and $\nabla^{T}=\left(\partial_{x}, \partial_{y},-k\right)$. The solutions of (13) are calculated by the Arnoldi method implemented in the Arpack library coupled with a LU inversion algorithm. This method provides a selected number of eigenvalues and the corresponding eigenvectors in the vicinity of a prescribed guess value. We performed several calculations with this method to span a significant part of the parameter space $(k, R e)$ in the low Reynolds $R e<3180$ and wavenumber region $k>0$. Owing to the fact that the matrices $A_{k, R e}$ and $B$ in Eq. (13) are real, $\lambda$ and its conjugate are companion solutions of (13).

All the modes are normalized to unit kinetic energy. Unsteady modes are rearranged such that their phase is zero at $\boldsymbol{x}=0$. This last operation does not change the results since Eq. (13) is unchanged when a phase shift is applied but enables an easier comparison between modes. To impose unit kinetic energy $\|\tilde{\boldsymbol{q}}\|=1$, we adopt the following scalar product:

$$
\left\langle\tilde{\boldsymbol{q}}_{1}, \tilde{\boldsymbol{q}}_{2}\right\rangle=\int \tilde{\boldsymbol{q}}_{1}^{T} B \tilde{\boldsymbol{q}}_{1} d \boldsymbol{x} .
$$

The matrices $A_{k, R e}$ and $B$ are built using the finite element code FreeFem $++\left(\right.$ see Hecht et al. ${ }^{16}$ ). Spatial discretization elements $\mathrm{P} 2$ and P1 are used for the velocity and the pressure, respectively. The numerical domain consists of a square centered on the vortex pair which has a side-length equal to $10 D$. A Dirichlet boundary condition $\boldsymbol{u}^{\prime}=0$ is specified at inflow and side frontiers and a usual outflow condition is set at the outlet. A convergence study was performed in terms of the size of the computational domain and of the spatial resolution to make sure that the results did not suffer from the influence of the boundaries (which induce image vorticity) and from numerical diffusion. In particular, no influence of the domain length behind the dipole was found. Finally, we verified that the 3D spectrum provided by Billant et al. ${ }^{2}$ was successfully retrieved by our method. The results of this comparison are given in Appendix B.

\section{3D STABILITY}

The complete spectrum of the LCD has been calculated for $R e=1280$. Figure 2 shows the spectra associated with modes S and A (see Subsection II A for the definition of these symmetries). We consider the normalized values of the growth rate and frequency, $\sigma^{*}$ and $f^{*}$ given by

$$
\sigma^{*}=\sigma \frac{D}{U}, \quad f^{*}=\omega \frac{D}{2 \pi U}
$$

The square symbols are white or black to indicate either nil or finite frequency. The modes found by Billant $e t a .^{2}$ are fully recovered. Modes that are not dominant are also found. This is an advantage 
(a)

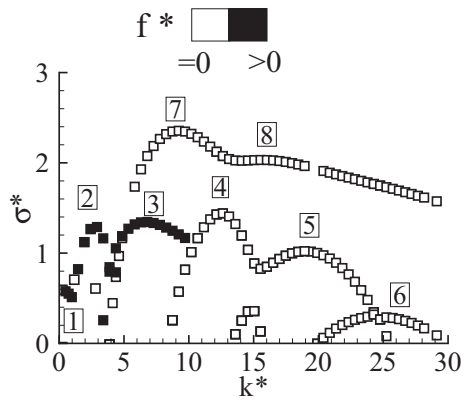

(b)

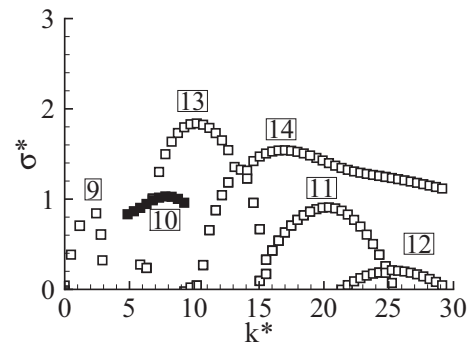

FIG. 2. Three-dimensional spectrum of the $\mathrm{LCD}$ at $R e=1280$. (a) A spectrum. (b) S spectrum. The black and white filling inside the square symbols indicate the finite or nil value of the normalized frequency.

of the method used for the calculation of the modes, that enables to look for eigenvalues that are not maximum. In comparison, Billant et al. ${ }^{2}$ used linearized simulations to calculate the unstable modes. This method limits the search to the most unstable modes (albeit further developments also enable the calculation of non-maximal modes). The spectra appear to be very rich when compared to the spectra obtained for lower aspect ratio dipoles (see Tsai and Widnall ${ }^{17}$ and Sipp and Jacquin, ${ }^{18}$ for instance). It is supposedly the large aspect ratio of the LCD that gives rise to this unstable dynamics which is continuously spred over the three-dimensional range of axial wavenumbers $k$.

In order to simplify the presentation, we have numbered the families of modes that appear in these spectra. Fourteen families are distinguished, each of them associated with a particular bump in Figure 2. Most of the modes are purely stationary, i.e., their frequency $f$ is nil. Only families $1,2,3$, and 10 exhibit non-stationary modes. In Figure 3, we present the vorticity fields associated with these 14 families. In each family, only one element is presented, corresponding to a specific wavenumber. Note also that only the real parts of the modes are shown.

The A modes 2, 7, 8 and the S modes 9, 10, 13, and 14 are those already found by Billant et al. ${ }^{2}$ Their shapes in Figure 3 are identical to those presented in Billant's ${ }^{2}$ paper. In particular, family 9 corresponds to the Crow instability, as can clearly be stated from the shape of the vorticity distribution $\left(\right.$ see $\left.\mathrm{Crow}^{5}\right)$. The other modes comprise sub-optimal modes which correspond to numbers 3, 4, 5, and 6 in case A and 11 and 12 in case S. Family 1 also appears new.

The vorticity distributions of these modes show the type of perturbations that are involved in these three-dimensional instabilities. In particular, all the modes yield Kelvin waves in the vortex cores with various radial and azimuthal patterns. Those are usually characterized by the number $n$ of nodes of the vorticity distribution in the radial direction and by the periodicity $m$ in the azimuthal direction. The stationary modes 4, 5, 6, 7, 8, 11, 12, 13, and 14 are all associated with the elliptical instability and have the characteristic structure of the Kelvin wave coupling $(m=-1$ and $m=1)$. Fragmentation of the vorticity field increases with the wavenumber in agreement with the increasing effect of viscous diffusion that acts as $k^{2}$ in the linearized equations. As a consequence of the
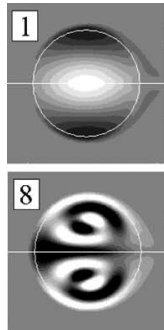
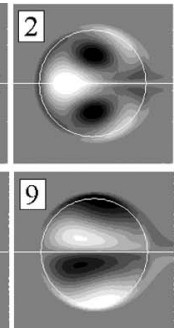
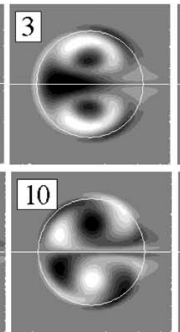
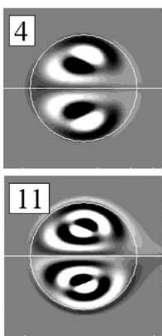
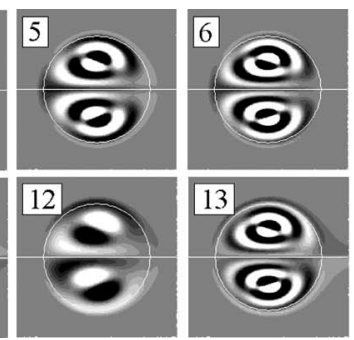

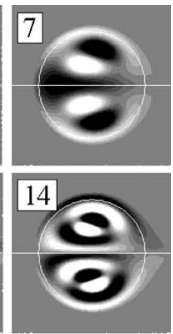

FIG. 3. Real parts of the vorticity fields $\zeta^{\prime}$ of the modes numbered from 1 to 14 in Figure 2. The Reynolds number is 1280. The modes are taken at the following wavenumbers: (1) $k^{*}=0.24$, (2) $k^{*}=2.9$, (3) $k^{*}=6.8$, (4) $k^{*}=12.6,(5) k^{*}=18.9$, (6) $k^{*}=25.2$, (7) $k^{*}=9.2$, (8) $k^{*}=15.5$, (9) $k^{*}=2.4$, (10) $k^{*}=5.3$, (11) $k^{*}=20.4$, (12) $k^{*}=10.2$, (13) $k^{*}=6.8$, (14) $k^{*}=17$. 
complete filling of the oval by the base flow vorticity, the modal vorticity is also distributed over the entire oval and even leaks outside of it.

In Secs. IV A and IV B, we will look successively into two specificities of the spectrum pertaining to the large-wavelength domain that have not been investigated before. The first point concerns the existence of a family of S modes that is not visible in Figure 2(b) because its extent in wavenumber space and amplification rate are too small (the presentation is done in Sec. IV A). The second point concerns the family numbered 1 in Figure 2(a) which comprises three-dimensional large wavelength modes.

\section{2D STABILITY}

\section{A. Mode S}

A new family of stationary and symmetric unstable modes was found in the large-wavelength limit, that we thereafter call mode $\mathrm{S}$ for convenience. The dependence of the growth rate upon the Reynolds number and wavenumber is explicated in Figure 4. The instability arises above the critical Reynolds number $R e_{c, S}=363$ and the most unstable mode is $2 \mathrm{D}$. The growth rate of the mode increases between $R e_{c, s}$ and $R e=1460$ and steadily decreases above. The instability is reduced for non-zero wavenumbers, i.e., when $3 \mathrm{D}$ effects take place. There is an associated cutoff which is equal to $k^{*} \simeq 2.8$ for $R e=1280$ used to calculate Figure 4(b).

The vorticity field associated with the 2D mode is shown in Figures 5(a) and 5(b). It features a real and an imaginary part that show exactly opposed shapes although one has to note that the imaginary part shown in Figure 5(b) has much lower vorticity levels than the real part. The same iso-contours have been used but the associated vorticity values have been increased by a factor 50 in the plot of the imaginary part. The mode is complex but the frequency is nil. The mode is thus stationary. The real and the imaginary parts correspond to two possible and opposite perturbation states.

The most prominent feature of the mode $\mathrm{S}$ is its $m=1$ azimuthal structure in the vortex cores, with $n=0$. Modes with $m=1$ and $n=0$ are known as displacement modes because they induce the displacement of the vortex as a whole. We also note that there is a quasi-symmetry of the mode about the (Oy) axis and that its structure remains unchanged when $k$ varies.

The mode features four tails of vorticity downstream of the dipole that leak from the positive and negative vorticity distributions attached to the dipole. The comparison between the circulations
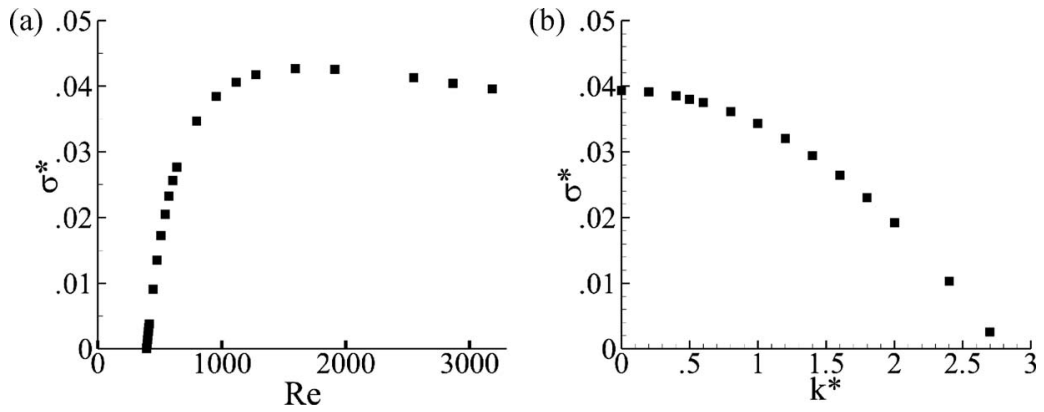

FIG. 4. Growth rate of mode S. (a) As a function of the Reynolds number. (b) As a function of the wavenumber at $R e=1280$ as in Figure 2.

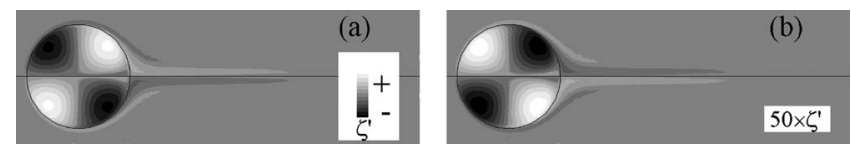

FIG. 5. Vorticity of mode $\mathrm{S}$ at $k=0$ and $R e=1280$. (a) Real part of the mode. (b) Imaginary part of the mode. In frame (b), the iso-contours are increased 50 times compared to frame (a). The levels of iso-contours have been adjusted in order to make apparent the tail of vorticity behind the dipole. 

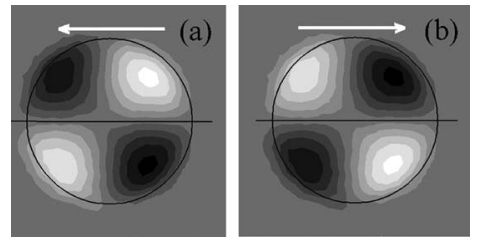

FIG. 6. Vorticity field obtained by artificially displacing the LCD dipole. (a) The dipole is displaced to the left. (b) The dipole is displaced to the right.

inside and outside the dipole, measured by $\Gamma_{\text {inside }}^{\prime}$ and $\Gamma_{\text {outside }}^{\prime}$ which are defined by

$$
\Gamma_{\text {inside }}^{\prime}=\int_{r<D / 2, y>0} \zeta^{\prime}(x, y, t) d x d y, \quad \Gamma_{\text {outside }}^{\prime}=\int_{r>D / 2, y>0} \zeta^{\prime}(x, y, t) d x d y
$$

shows that the perturbation total circulation $\Gamma^{\prime}$ is equal to $\Gamma_{\text {outside }}^{\prime}+\Gamma_{\text {inside }}^{\prime}=0$. The integrals are calculated only in upper domain $y>0$ so that the symmetry effect is removed. As will be discussed later (see Sec. V), total circulation is a conserved quantity, and this is also true at perturbation level. This necessarily imposes $\Gamma^{\prime}=0$. An interpretation of the effect of mode $S$ on the flow is obtained by considering the sum of the vorticity of the mode and that of the base flow displayed in Figure 1. Doing so indicates that mode $\mathrm{S}$ leads to a shift of the dipole to the left (real part) or to the right (imaginary part). This view is confirmed by considering a small displacement of the base flow to the left and to the right. The modifications to the flow implied by this displacement are shown in Figure 6 and agree well with the vorticity distribution of the unstable mode.

\section{B. Mode A}

The spectrum associated with family 1 is shown in Figure 7. What we now call mode A makes the dipole unstable to large wavelength perturbations when the Reynolds number is larger than $R e_{c, A}=22$. This critical Reynolds number is lower than for mode $\mathrm{S}$. Mode $\mathrm{A}$ is strongest for $k=0$ and exhibits a maximum at $R e=223$. This maximum growth rate equals $\sigma^{*}=0.69$. The growth rate can also be evaluated in units of the induced strain rate $\gamma=\Gamma / 2 \pi b^{2}$. The calculation yields $2 \pi b^{2} / \Gamma \simeq 0.46(D / U)$ so that $\max (\sigma)=0.69[U / D]=0.32\left[\Gamma /\left(2 \pi b^{2}\right)\right]$.

Figure 8 shows the vorticity field of the most unstable mode $(k=0$ and $R e=223)$. The real and imaginary parts of the mode represent the flow at a $\pi / 2$ phase interval. They both feature displacement modes inside the dipole that merge at the mid-plane.

Like mode S, mode A features a wake downstream of the dipole. This wake is composed of alternating positive and negative vorticity regions along the (Ox) direction. The conservation of total circulation of the flow, which is nil initially (i.e., for the baseflow) imposes that total perturbation

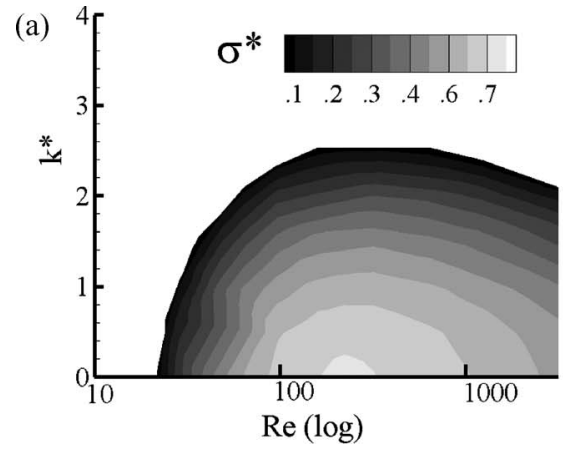

(b)

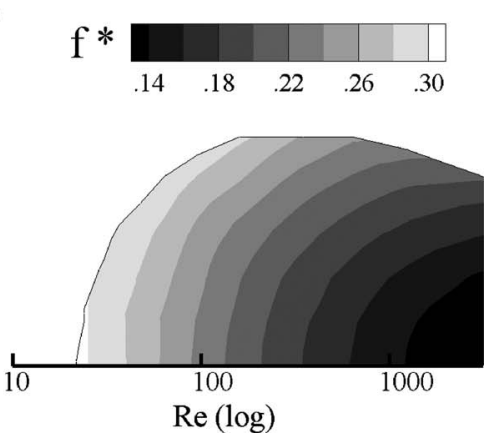

FIG. 7. Domain of instability of the LCD featuring the normalized growth rate $\sigma^{*}=\sigma D / U$ in frame (a) and the normalized frequency $f^{*}=\omega D /(2 \pi U)$ in frame (b) as a function of $k^{*}$ and $R e$. 


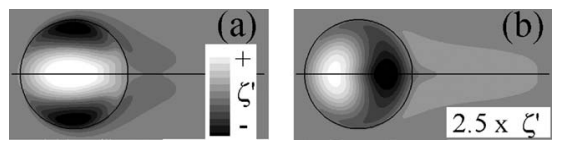

FIG. 8. Perturbation vorticity of mode $\mathrm{A}$ at $k=0$ and $R e=223$. (a) Real part of the mode. (b) Imaginary part.

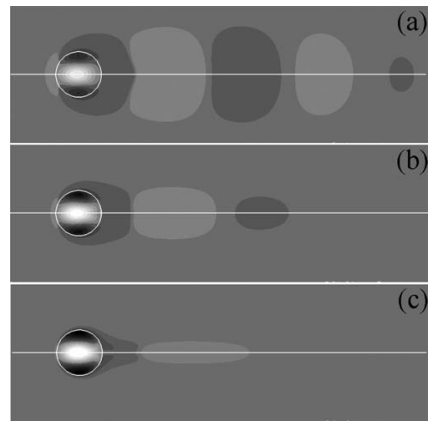

FIG. 9. Vorticity field of the unstable mode A for three values of the Reynolds number: (a) $R e=32$, (b) $R e=96$, (c) $R e=478$. The same iso-values have been used in the three frames. A low iso-value has been added to highlight the wake downstream of the dipole. The dipole and the symmetry line are indicated by the white lines.

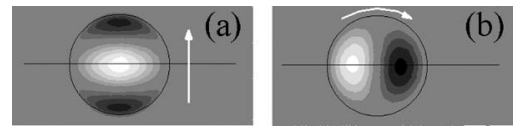

FIG. 10. Vorticity field obtained by artificially moving the LCD. (a) Effect of an upward translation. (b) Effect of a clockwise rotation.

circulation $\Gamma^{\prime}=\Gamma_{\text {inside }}^{\prime}+\Gamma_{\text {outside }}^{\prime}=0$, with these circulations defined in (17). The calculation of these circulations with the modal vorticity field shows that this is indeed the case.

The effect of viscosity on the wake is illustrated in Figure 9 which shows the real part of the vorticity field associated to mode A for three different Reynolds numbers. Since the wake contains vorticity levels much lower than those present in the oval, an iso-contour with a low value of vorticity is added in the uniformly distributed set of iso-values. While the part of the mode in the oval shows almost no variation as a function of the Reynolds number, except a lowering of the vorticity magnitude, the part of the mode outside the oval seems to extend further as the Reynolds is decreased. For instance, the vertical extent of the wake is seen to increase greatly with the Reynolds number, being large at $R e=32$ which is just above the threshold of instability (frame (a)) and narrow when the Reynolds number is an order of magnitude larger (frame (c)). In the same way, the upstream part of the perturbation vorticity protrudes further upstream as the viscosity is increased. Using the entire data set we find that the spatial extension of the perturbation vorticity field, taking, for instance, the width of the vorticity wake as a measure of this extension (taken as the vertical extent of the perturbation vorticity field at a given location sufficiently downstream of the dipole), scales as $R e^{-1 / 2}$.

The vorticity distribution of mode A can be interpreted by superposing the perturbation and the base flow vorticity fields (using Figures 1 and 8 ). The displacement modes in the state shown in Figure 8(a) induce the displacement of both vortices in the upward direction. In Figure 8(b), the upper and lower vortices are moved to the upper right and lower left, respectively. Therefore, the real part of the mode corresponds to an upward displacement of the dipole and the imaginary part corresponds to a clockwise rotation. In these movements, the dipole is moved almost as a whole. To confirm this, we calculated the modification of the base flow when it is subjected to (i) a solid upward translation and (ii) a solid clockwise rotation. The results, shown in Figure 10 agree well with Figure 8, and confirm the scenario. 


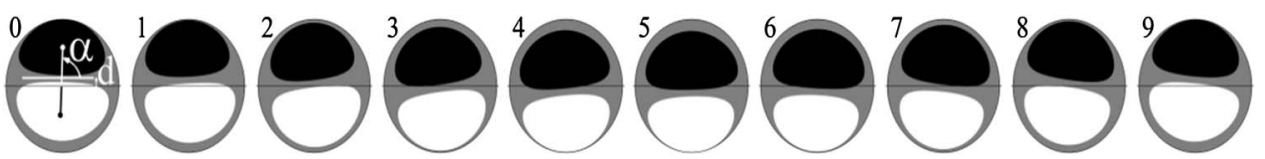

FIG. 11. Time sequence of mode A during one period of oscillation at $R e=223$ and $k=0$. The frames show the total vorticity field $\zeta$ at different times corresponding to $10 \times t / T_{A}$. In the first frame, $\alpha$ and $d$ denote the angle of the displacement and the vertical displacement of the dipole.

\section{Linear evolution of mode A in 2D}

This section is devoted to the description of the unsteadiness associated with mode $\mathrm{A}$ at $k=0$. Figure 11 shows the time sequence of the total vorticity $\zeta(x, y, t)$ composed of the base flow vorticity $\bar{\zeta}(x, y)$ and of the most unstable mode shown in Figure 8, i.e.,

$$
\zeta(x, y, t)=\bar{\zeta}(x, y)+\epsilon \zeta^{\prime}(x, y, t)
$$

with the value of $\epsilon$ chosen arbitrarily large to highlight the dipole oscillations. Figure 12 shows the simultaneous evolution of the sole perturbation vorticity $\zeta^{\prime}(x, y, t)$. In these time sequences, the time frame covers the full oscillation period of mode A, i.e., $T_{A}=2 \pi / \omega_{A}$ at $R e=223$ and $k=0$, with a time step equal to $0.1 T_{A}$. Initially, the flow is in the state corresponding to the real part of the mode shown in Figure 8(a). The imaginary part of the mode shown in Figure 8(b) appears between steps 2 and 3. In Figure 11, only two levels of iso-contours were used in order to simplify the structure of the flow. The amplification factor has also been turned off to make apparent the kinematics of the instability alone.

The time evolution helps to understand how the translation and rotation movements previously identified act together. We monitor the rotation of the dipole by the tilt angle $\alpha$ schematized in the first frame of Figure 11. This angle measures the orientation of the line linking the vortex centers about the horizontal axis. The translation of the dipole is monitored by its vertical position $d$. In frame 0 , the dipole is shifted upward $(d>0$ ) and is almost horizontal (i.e., $\alpha \simeq \pi / 2$ ). The dipole then starts to rotate counter-clockwise. At the same time, a downward motion begins which lasts up to step 5. Rotation stops between steps 3 and 4, and reverses to start a clockwise movement in the following time steps. We then observe the same kinematics but with opposite directions: the upward motion follows the clockwise rotation, and the kinematics goes back to step 0. In terms of perturbation vorticity, the time sequence in Figure 12 shows that the dipole oscillations are associated with the countergrade rotation of the displacement modes in each vortex core. In addition, looking simultaneously at Figures 11 and 12 shows that the clockwise rotation of the dipole leads to the shedding of positive vorticity (and therefore counter-clockwise rotation is associated with negative vorticity). This phenomenon is coherent with the conservation of total circulation in the flow $\Gamma_{\text {outside }}^{\prime}=-\Gamma_{\text {inside }}^{\prime}$ that was mentioned previously.

This description leads us to interpret the up and down movements of the dipole as a consequence of the orientation $\alpha$. Remember that the dipole is self-propelled to the left as a consequence of its own induction. Therefore, when the dipole tilts, it changes the orientation of the propagation velocity vector: upward for $\alpha<\pi / 2$ and downward otherwise. In the reference frame attached to the base flow, this modification takes the form of almost purely vertical movements. Figure 13(a) shows the
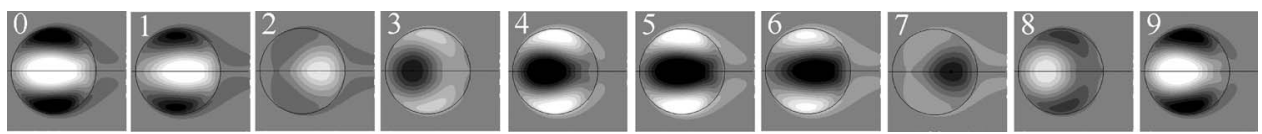

FIG. 12. Same as Figure 11, but showing the evolution of the perturbation axial vorticity $\zeta^{\prime}$. The grey scale is chosen identical to that in Figure 8. 


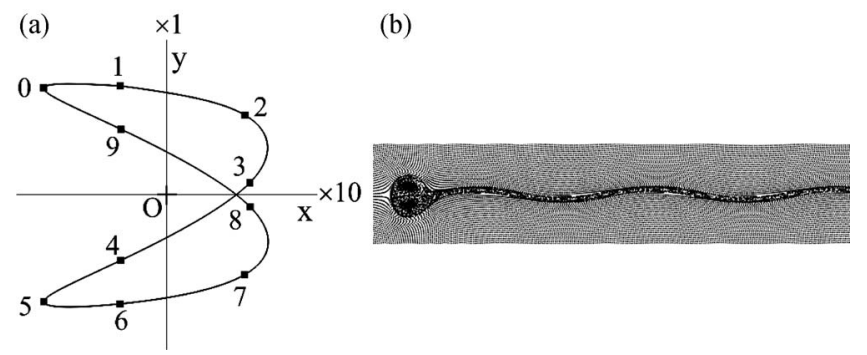

FIG. 13. Motion of the dipole destabilized by mode A. (a) Motion in the reference frame attached to the base flow. (b) Motion in the laboratory reference frame illustrated by streaklines. Frame (a) shows the evolution of the point $M\left(x_{d}, y_{d}\right)$ defined in (19) during one period of motion.

trajectory of the dipole center $\boldsymbol{x}_{d}=\left(x_{d}, y_{d}\right)$ defined by

$$
\boldsymbol{x}_{d}=\frac{\int_{r<D / 2}|\zeta(x, y, t)| \boldsymbol{x} d S}{\int_{r<D / 2}|\zeta(x, y, t)| d S} .
$$

This relation defines the barycentre of the absolute value of the axial vorticity contained inside the Kelvin oval. The pattern represents a figure eight. However, it should be noted that the motion is essentially vertical since the horizontal axis is magnified 10 times. The forward and backward movements also result from the modification of the orientation of the trajectory. Seen in the laboratory reference frame, the instability amounts to zigzag oscillations of the dipole. Figure 13(b) shows the streaklines of the flow which highlight the form of the wake shed downstream of the dipole as it oscillates. The streaklines were obtained numerically by calculating the paths of several particles initially located along a vertical line 5D ahead of the dipole and inside the dipole $(\epsilon=1$ is chosen for the amplitude of perturbation).

\section{ANALYSIS OF THE INSTABILITIES}

\section{A. Impulse and circulation}

The fact that the dipole is displaced by the instabilities points out the need to analyse the consequences of the conservation laws for impulse and circulation. Recall that the impulse is defined as the first moment of the vorticity field

$$
\boldsymbol{I}=\int_{V} \boldsymbol{x} \times \omega d V
$$

and according to Saffman ${ }^{3}$

$$
\frac{d \boldsymbol{I}}{d t}=\int_{V} \boldsymbol{F} d V
$$

where $\boldsymbol{F}$ are the non-conservative external body forces. Since in the present case these forces are absent the impulse is an invariant of the flow. Considering the circulation $\Gamma$ of the dipole vortices and their separation $b$, the impulse of the undisturbed dipole is equal to $\boldsymbol{I}=-\Gamma b \boldsymbol{e}_{x}$. This impulse corresponds to the displacement of the dipole to the left at a steady velocity $U$ in the laboratory reference frame, as has already been mentioned. It is good to keep in mind that conservation of impulse is the vortex equivalent to conservation of momentum (see $\operatorname{Saffman}^{3}$ ). Linearizing relation (20) yields the perturbation impulse $I^{\prime}=\int_{V} x \times \omega^{\prime} d V$ which must be nil at all times. It is straightforward to show that conservation of the x-impulse concerns only $\mathrm{S}$ perturbations, while A perturbations are concerned with the conservation of the y-impulse. The 2D perturbation impulse is thus expressed as $\boldsymbol{I}^{\prime}=\left(I_{S}^{\prime}, I_{A}^{\prime}\right)$ with $I_{S}^{\prime}, I_{A}^{\prime}=0$.

The second law of conservation - that of circulation - states that the total circulation of the flow is a conserved quantity, including viscous diffusion if the closed contour is taken large enough. Since 
the baseflow is made of balanced vortices of circulation $\pm \Gamma$ the total baseflow circulation is nil. At the perturbation level, considering A and S perturbations separately, the perturbation circulation $\Gamma^{\prime}$ is an invariant of the half-domains ( $y$ positive or negative) and must remain nil.

Generally speaking, dipole motion necessarily implies variation of impulse. However, this is impossible in the absence of an external forcing if in return no other impulse is brought into the flow to balance this variation. The analysis of $\mathrm{Crow}^{5}$ on the stability of a pair of vortex filaments revealed no unstable mode in $2 \mathrm{D}$, which is fully consistent with this analysis. In the present case however, there appears to be additional vorticity in the flow - at the perturbation level - that permits such a balance and thus the dipole movements.

The perturbation process is achieved at constant perturbation circulation $\Gamma^{\prime}=0$ and this has been verified in Secs. IV A and IV B. This means that the perturbation vorticity attached to the wake (outside the dipole) is exactly balanced by the perturbation vorticity present inside the dipole. We mentioned earlier when analysing the time sequence in Figure 11 that the (counter-) clockwise rotation of the dipole leads to the shedding of (negative) positive vorticity. This agrees with the conservation of circulation.

\section{B. Vortex dynamics}

The perturbation vorticity fields of mode $\mathrm{S}$ and $\mathrm{A}$ in Figures 5 and 8 yield three different vorticity zones: the one (i) associated to the displacement modes, that (ii) associated to the wake and that (iii) present inside the dipole but different from the displacement modes, such as, in the case of mode $\mathrm{S}$, the perturbation vorticity present above and below the symmetry line in the region near the downstream stagnation point. A better understanding of how these different vorticity zones are generated their interactions is essential to analyse the instabilities. All the processes at play are contained in the equation for the perturbation vorticity $\zeta^{\prime}$ in 2D which writes

$$
\frac{\partial \zeta^{\prime}}{\partial t}+(\overline{\boldsymbol{u}} \cdot \nabla) \zeta^{\prime}=-\left(\boldsymbol{u}^{\prime} \cdot \nabla\right) \bar{\zeta}+R e^{-1} \Delta \zeta^{\prime}
$$

The LHS of this equation contains the time derivative of the perturbation vorticity and its transport by the baseflow. In the RHS, the first term $\left(\boldsymbol{u}^{\prime} \cdot \nabla\right) \zeta$ corresponds to the transport of the basic vortices by the perturbation. A useful point of view is to see the perturbation velocity as being induced by the perturbation vorticity and thus to write the Biot and Savart law at the perturbation order which relates $\zeta^{\prime}$ to $\boldsymbol{u}^{\prime}$

$$
\boldsymbol{u}^{\prime}(x, y)=\frac{1}{2 \pi} \int_{V} \frac{\left(x-x^{\prime}\right) \times\left(\zeta^{\prime}\left(x^{\prime}, y^{\prime}\right) \boldsymbol{e}_{z}\right)}{\left(\left(x-x^{\prime}\right)^{2}-\left(y-y^{\prime}\right)^{2}\right)} d x^{\prime} d y^{\prime} .
$$

The linearity of the Biot and Savart law legitimates its use for the analysis of the perturbative flow. It allows simple reasoning based on the induction effect of the vorticity field as will be done below. Finally, the last term of (22) is the viscous diffusion of the perturbation vorticity.

The importance of the displacement modes (i) in the present instabilities justifies that we first recall their dynamics in the simpler case of an isolated vortex. A short review on the displacement modes indicates, see Saffman ${ }^{3}$ and Fabre et al. ${ }^{19}$ that a displacement mode has a general shape of a well balanced dipole superimposed onto the vortex. At $k=0$, the displacement mode is stationary. At finite $k$ it is retrograde. In 2D, the stationarity of the displacement mode results from the equilibrium between the convection due to the baseflow and that due to the perturbation, i.e., the term $(\overline{\boldsymbol{u}} \cdot \nabla) \zeta^{\prime}$ in Eq. (22) balances the term $\left(\boldsymbol{u}^{\prime} \cdot \nabla\right) \bar{\zeta}$. The vorticity field of the displacement mode of a single vortex is illustrated in Figure 14. In the depicted situation, the vortex is displaced upward, since the lobes of the displacement mode are stacked vertically and the upper lobe holds negative vorticity like the vortex.

Compared to the single vortex case, the stationarity of the displacement modes is not observed in the case of mode A. Figure 12 shows that they rotate in the direction opposite to the flow. They do not have either the well-balanced shape which is depicted in Figure 14, the lobe on the symmetry line of the dipole being always larger than the other lobe. These differences compared to the single vortex case need to be explained. 


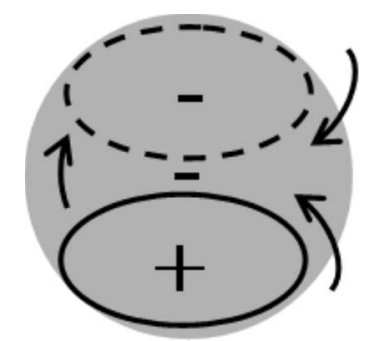

FIG. 14. Form of the displacement mode at $k=0$ on a single vortex. The vortex taken of negative vorticity is shown by a grey disc. The displacement mode is shown by a perturbation dipole in plain and dotted lines depending on the sign of the associated vorticity (indicated by the curved arrows).

Concerning the unbalance between the two lobes of the displacement mode in each vortex, the time sequence of mode A in Figure 12 suggests that such a disequilibrium results from a loss of vorticity through the oval boundary. The mechanism of this loss can be elucidated as follows. Outside the oval and due to the absence of baseflow vorticity equation (22) reduces to $\frac{d \zeta^{\prime}}{d t}=R e^{-1} \Delta \zeta^{\prime}$ meaning that perturbation vorticity can be present only if it is diffused by viscosity or if it is already present. The convective flow regime outside the oval and the upstream flow which is free of any vorticity excludes the presence of perturbation vorticity outside the oval and thus only viscous diffusion of the vorticity present inside the oval can participate to the wake. The scaling of the wake size on the Reynolds number $\left(\propto R e^{-1 / 2}\right)$ in fact already suggested this scenario. In addition, the perturbation vorticity fields exhibited by the smooth dipole shown in Figures 23 and 24 of Appendix A, which exhibit finite levels up to the oval boundary, also agree with this conclusion since in this case too, the perturbation vorticity of the displacement modes can leak outside the oval despite the more compact baseflow vorticity. It thus appears that the transfer of vorticity outside the oval is enabled by the large extension of the displacement modes inside the oval, quite regardless of the potentially limited spatial extent of the baseflow vorticity.

The rotation of the displacement mode in mode A is to be understood thanks to the breaking of symmetry of the displacement mode. This is illustrated in the schematic (Figure 15) where only the case of the upper vortex has been considered, the results being equally applicable to the lower vortex. Initially (frame (a)), the displacement mode superimposed onto the vortex is well-balanced, as in Figure 14. However, unlike the single vortex case depicted in Figure 14, loss of vorticity occurs at the oval boundary as illustrated by the array of arrows. As a result (frame (b)), the upper lobe becomes weaker than the lower lobe. In return, according to the Biot and Savart law (23) and the term $\left(\boldsymbol{u}^{\prime} \cdot \nabla\right) \bar{\zeta}$ which refers to the convection of the basic vorticity by the perturbation flow field (applied at the vortex center), the induction effect of the lower lobe vorticity on the vortex makes it move toward the left, as illustrated by the thick arrow starting from the vortex center in frame (c). Since the vortex is initially in an upward position, the leftward motion amounts to a counter-clockwise rotation of the displacement mode. The opposite configuration with the displacement mode oriented upside down leads to the same conclusion. In this case, the perturbation velocity $\boldsymbol{u}^{\prime}$ induced by the lower

(a)

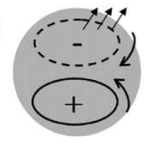

(b)

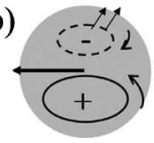

(c)

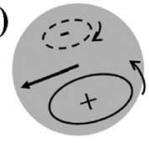

(d)

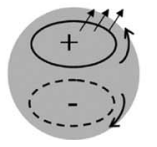

(e)

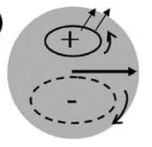

(f)

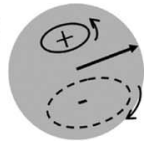

FIG. 15. Explanation of the rotation of the displacement mode in a vortex subjected to a localized action of viscosity shown by the arrays of arrows. The vortex is shown in grey and the loss of vorticity through the oval boundary by the array of arrows. The curved arrow indicates the rotation of the vorticity zones. The initially well-balanced vortex dipole of perturbation in (a) loses vorticity at the oval boundary. This results in frame (b) in a relative strengthening of the lower lobe compared to the upper one. This leads, through the term $\left(\boldsymbol{u}^{\prime} \cdot \nabla\right) \bar{\zeta}$ of Eq. (22), to the modification of the vortex position, initially in an upward position, such that the displacement mode rotates as shown in frame (c). The induced velocity $\boldsymbol{u}^{\prime}$ is shown by the arrow on the vortex. The case with the displacement mode the other way around in frames (d), (e), and (f) evolves in the same way. 


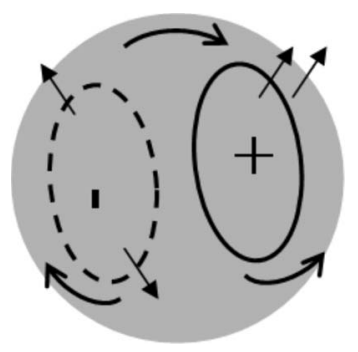

FIG. 16. Effect of vorticity loss on the displacement modes in mode S. This is a schematic of the upper vortex in the case of the real part of mode $\mathrm{S}$. The arrows indicate the loss of vorticity to the outside of the oval.

lobe (which dominates over the upper lobe) makes the vortex move to the right which, the vortex being initially in a downward position, amounts to a counter-clockwise rotation like previously. This mechanism of displacement mode unbalance induced by the vorticity loss at the oval boundary permanently maintains the displacement mode into a countergrade rotation in the upper and lower vortices, exactly as what is observed in the time sequence in Figure 12.

The scenario is different in the case of mode $S$ because the leak of vorticity occurs almost equally for the right and the left lobes of the displacement mode. This is illustrated in Figure 16. A close look at the orientation of the displacement modes in mode $\mathrm{S}$ shows that they are slightly inclined, which results in vorticity being lost at the upper and lower boundaries in the left lobe but only at the upper boundary in the right lobe. Since the two lobes of the displacement are equally depleted, the displacement mode remains stationary.

While the kinematics of the dipole is better understood with the previous analysis, the ingredients for the unstable dynamics of the $\mathrm{S}$ and A modes are still lacking. To become unstable and maintain the kinematics implied by mode $\mathrm{A}$ and $\mathrm{S}$, the dipole needs to gain momentum. Two mechanisms for this are highlighted below, (i) the effect of the mutual strain field and (ii) the contribution of the wake.

\section{Effect of the mutual strain field}

The effect of the mutual strain field in 2D as discussed in the Introduction implies that the orientation of the displacement of the vortices of the dipole is an important parameter for the instabilities. Recall that, as described by Moore and Saffman ${ }^{8}$ and Robinson and Saffman, ${ }^{9}$ a single vortex embedded in an external strain field is unstable. In such a strain field, the perturbation to the single vortex takes the form of a displacement mode which moves unboundedly in the positive axis of strain. This result can be used to analyse the present configuration since the imposed strain field models the role of the mutual strain field between the vortices of the pair. Figure 17 shows a schematic of the proper directions associated with this mutual strain field. The rotation of the flow inside the dipole, that determines the orientations of the arrows, is chosen identical to that of the baseflow in Figure 1. The axis are oriented at $+45^{\circ}$ and $-45^{\circ}$ in the top vortex and symmetrically

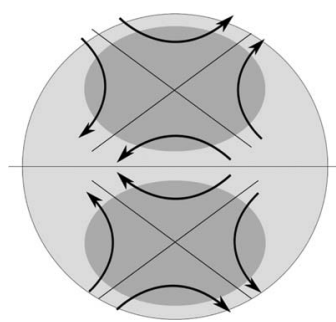

FIG. 17. Enlarged view of the strain field induced between the vortices of the pair in the vicinity of the vortex centers. Note that the strain field further from the vortex centers is not indicated. 


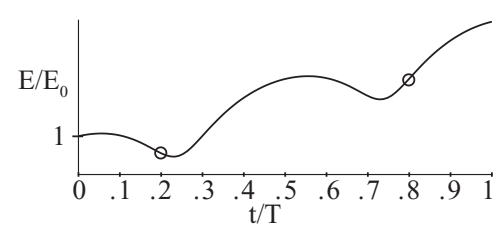

FIG. 18. Evolution of the kinetic energy of mode $\mathrm{A}$ as a function of time for $k=0$ and $R e=223$. The two circles correspond to the selected snapshots 2 and 8 in Figure 12.

in the bottom vortex. Note that for 3D perturbations, this strain is the driving mechanism of the instabilities such as the so-called long and short-wave instabilities. ${ }^{5,20}$

A first observation of the effect of this strain is the temporal evolution of the perturbation kinetic energy $E(t)$ associated with mode $\mathrm{A}$ at $k=0$, the kinetic energy being defined as follows:

$$
E(t)=\int \frac{1}{2}\left\|\boldsymbol{u}^{\prime}\right\|^{2} d \boldsymbol{x}
$$

where the integral is over the entire computational domain. This evolution, normalized by the initial energy $E_{0}=E(0)$, is shown in Figure 18 with the same time reference as the time sequence in Figure 11. The most noticeable feature is the periodic increase of energy. In Figure 12, the snapshots 2 and 8 show the perturbation axial vorticity at $t=0.2 T_{A}$ and $t=0.8 T_{A}$ when the energy decreases and increases, respectively. Using the schematic in Figure 17, one can see that at $t=0.2 T_{A}$ the displacement modes are oriented in the direction of compression while at $t=0.8 T_{A}$ the displacement modes are oriented in the direction of stretching. This close match between the transient growth of the perturbation mode and the orientation of the displacement modes shows that the strain field plays the role of a transient amplifier in the evolution of mode A.

This transient growth also impacts the viscous leaking of vorticity that was analysed before. With the increase of the displacement mode when it is in the direction of positive strain, viscous diffusion at the dipole boundary is also increased. Oppositely when the displacement modes are in the direction of compression, viscous leaking is almost stopped, as is observed in Figure 12 (see, for instance, steps 1,2, and 3). The periods of growth of the displacement modes are thus characterized by an important shedding of vorticity in the wake while those of decrease result in a sudden stop of the shedding.

Concerning mode $\mathrm{S}$, the effect of the mutual strain field is not as obvious as for mode $\mathrm{A}$. The orientation of the displacement modes in mode $\mathrm{S}$ is only slightly oriented along the direction of positive strain (an angle of approximately $1.3^{\circ}$ is measured from Figure 5), suggesting that only a part of the growth of mode $\mathrm{S}$ could be explained by this mechanism.

\section{Contribution of the wake}

The last ingredient in the dynamics of the instabilities results from the motion of the dipole induced by the wake. This induction effect corresponds to the first term $-\left(\boldsymbol{u}^{\prime} \cdot \nabla\right) \bar{\zeta}$ on the RHS of Eq. (22). In order to evaluate the influence of the wake, we consider the velocity $\boldsymbol{u}^{i}$ induced by the wake upon the flow particles present inside the dipole. This induced velocity is obtained by restricting the Biot and Savart law (23) integration domain to the outside region of the dipole, yielding

$$
\boldsymbol{u}^{i}(x, y)=\frac{1}{2 \pi} \int_{r^{\prime}>D / 2} \frac{\left(x-x^{\prime}\right) \times \zeta^{\prime}\left(x^{\prime}, y^{\prime}\right) \boldsymbol{e}_{z}}{\left(\left(x-x^{\prime}\right)^{2}-\left(y-y^{\prime}\right)^{2}\right)} d x^{\prime} d y^{\prime}
$$

This relation expresses the non-local effect of the perturbation vorticity present in the wake. Owing to the modal decomposition of the perturbation, this induced velocity can be expressed in terms of a modal quantity $\tilde{\boldsymbol{u}}^{i}$. Figure 19 shows the real and imaginary parts of $\tilde{\boldsymbol{u}}^{i}$ associated with mode $\mathrm{A}$ and $\mathrm{S}$ at a selected number $N$ of points inside the dipole. 
(a)

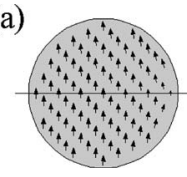

(b)

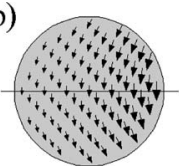

(c)

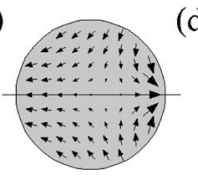

(d)

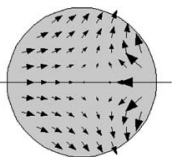

FIG. 19. Velocity field $\tilde{\boldsymbol{u}}^{i}$ induced by the wake vorticity inside the dipole for mode $\mathrm{A}(k=0$ and $R e=223)$ and mode $\mathrm{S}$ ( $k=0, R e=1280)$. (a) Real part of mode A. (b) Imaginary part of mode A. (c) Real part of mode S. (c) Imaginary part of mode $S$. Note that the same scaling has been used for setting the vector length in frames (a) and (b) while in frames (d) the vector length has been increased ten times compared to frame (c) to make the vectors visible.

The induced velocity at these locations is a part of the total perturbation velocity $\tilde{\boldsymbol{u}}$. One may accordingly write $\tilde{\boldsymbol{u}}=\tilde{\boldsymbol{u}}^{i}+\tilde{\boldsymbol{u}}^{\text {self }}$ with the second velocity field being that due to the perturbation vorticity already present inside the dipole upon itself. The importance of the wake effect upon the dipole dynamics can be evaluated thanks to the linear momentum associated with the velocity field shown in Figure 19. Then the induced linear momentum must be compared with the linear momentum associated with the flow present inside the dipole.

In the following, we thus consider the linear momentum $\tilde{\boldsymbol{L}}$ of the flow present inside the dipole and the induced linear momentum $\tilde{\boldsymbol{L}}^{i}$ due to the wake. The linear momentum is obtained by the following integration performed over the volume of the dipole, the outside field being excluded:

$$
\tilde{\boldsymbol{L}}=\int_{r<D / 2} \tilde{\boldsymbol{u}}(x, y) d \boldsymbol{x} \simeq \frac{\pi D^{2}}{4 N} \sum_{j=1}^{N} \tilde{\boldsymbol{u}}\left(x_{j}, y_{j}\right) .
$$

For $\tilde{\boldsymbol{L}}^{i}, \tilde{\boldsymbol{u}}$ is replaced by $\tilde{\boldsymbol{u}}^{i}$. The use of the Biot and Savart law to calculate the induced velocity field precludes the calculation of $\tilde{\boldsymbol{u}}^{i}$ at all the degrees of freedom of the computational field. Therefore, the integral in (26) for $\tilde{\boldsymbol{L}}^{i}$ is reduced to a sum over the $N$ points and $N$ is chosen large enough for convergence ( $N=70$ in practice). The total linear momentum $\tilde{\boldsymbol{L}}$ is calculated from the direct integration of the velocity fields of mode $\mathrm{S}$ and A shown in Figures 5 and 8. It has then been used as a reference to validate the integration over the $N$ points done in the right side of Eq. (26).

In the case of mode $\mathrm{A}$, the symmetry of the mode implies that the horizontal momentum of disturbance is nil. The calculation of the vertical momentum yields $\left|\tilde{L}_{y}^{i}\right|=0.32\left|\tilde{L}_{y}\right|$. The amplitude of the induction effect is thus of the same order as the total momentum contained inside the disturbed dipole. Moreover, there is a quarter period phase difference between the two quantities: the wake induction is delayed by $\Delta t=0.25 T_{A}$ compared to the dipole dynamics. This phase difference shows that there are periods of time when the wake induction plays in the same direction as the dipole motion and therefore increases it. Furthermore, due to this phase difference, the wake induced vertical motion is maximum when mode $\mathrm{A}$ is in the situation of its imaginary part which, on the basis of the time sequence in Figure 12, also suggests that the wake induction maintains the vertical position of the dipole at the times when the perturbation starts to rotate and to lose energy.

In the case of mode $S$, the symmetry of the mode implies that the vertical momentum of disturbance is nil. The calculation of the horizontal momentum yields $\tilde{L}_{x}^{i} \simeq-10 \tilde{L}_{x}$ with $\tilde{L}_{x}>0$ for the real part of the mode shown in Figure 5(a) and $\tilde{L}_{x}<0$ for the imaginary part. The total linear momentum is small compared to that induced by the wake. This suggests that the effect of the wake upon the dipole is almost entirely balanced by the self-induction of the perturbation vorticity present inside the dipole. Importantly, the wake induces the displacement of the dipole in the same direction as the displacement of the vortices which was analysed earlier (to the left in the case of the real part in Figure 5). Also the induced velocity field shows, at the vortex centers, vectors associated with $\tilde{\boldsymbol{u}}^{i}$ which are aligned with the direction of positive strain. Therefore in these regions, the wake pushes the vortices in the destabilizing direction. 


\section{DISCUSSION AND CONCLUDING REMARKS}

In this article, we have analysed the stability of the LCD in the long wavelength limit and, upon finding new instability modes whose maxima are purely 2D, we focused on the properties of the associated 2D instabilities, namely, the modes we referred to as $\mathrm{S}$ and $\mathrm{A}$ in relation to their symmetry. Mode $\mathrm{S}$ is a stationary mode with a weak growth rate. The associated instability takes the form of displacement modes in the vortices of the dipole, that move the dipole either upstream or downstream. Mode A is an unsteady mode with a quite significant growth rate, although it is not as strong as the 3D instabilities which are known to occur in the LCD. It also takes the form of displacement modes in the vortices of the dipole, the kinematics of which creates the oscillations of the dipole about its initially straight trajectory. The larger growth rate of mode A makes it the most interesting feature of the LCD two-dimensional dynamics.

The mechanisms of these instabilities have been revealed by an analysis of the perturbation vorticity field with the help of the perturbation vorticity equation and by invoking the necessary conservation of circulation and impulse. The fundamental ingredient of these $2 \mathrm{D}$ instabilities is the presence of perturbation vorticity outside the baseflow vortices, in the form of a wake. This wake enables the movement of the dipole vortices. It also contributes to the dipole motion and increases it. The increased dipole motion in return amplifies the wake and therefore we suggest that this forms the loop of amplification associated to the present instabilities. Another contributor to the instability was found to be the mutual strain field between the vortices. This amplifies the displacement of the vortices when they are in the axis of positive strain.

The time scale analysis that we performed in Subsection II B must be reexamined with the calculated complex frequency $\lambda$ of modes $S$ and $A$. In particular, the perturbation time scale considered in the beginning of this article was that of 3D perturbations whose instabilities are driven by the mutual strain $\gamma$ defined in (3). While the mutual strain impacts the instabilities, it is not the leading effect, and the perturbation time scale analysis must be repeated based on the calculated frequency of the unstable modes

$$
t_{\text {perturbation }}=\left\{\begin{array}{cl}
\frac{D}{U \sigma^{*}} & \text { if } f^{*}=0, \\
\frac{D}{U\left|\min \left(\sigma^{*}, f^{*}\right)\right|} & \text { if }\left|f^{*}\right|>0 .
\end{array}\right.
$$

Here, $t_{\text {perturbation }}$ is given in physical units. The ratio $r_{t}=t_{\text {base }} / t_{\text {perturbation }}$ then yields

$$
r_{t}=4 \operatorname{Re}\left|\min \left(\sigma^{*}, f^{*} \neq 0\right)\right|\left(\frac{a}{b}\right)^{2} .
$$

The minimum between the growth rate $\sigma^{*}$ and the frequency $f^{*}$ determines the slowest perturbation time scale to be considered when making comparisons against the baseflow evolution rate. Figure 20 shows the calculated value of $r_{t}$ for the $\mathrm{S}$ and A instabilities as a function of the Reynolds number. In the low range of Reynolds number in each instability case, the value of $r_{t}<10$ renders

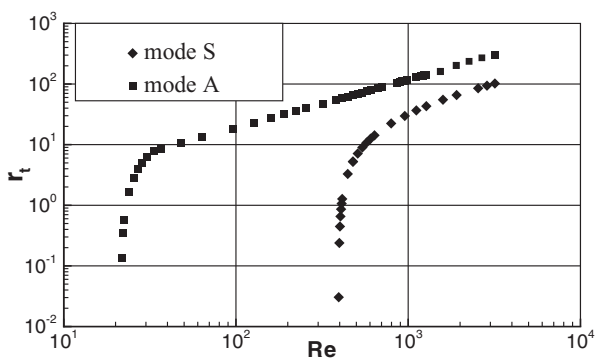

FIG. 20. Influence of the Reynolds number on the frozen baseflow hypothesis. The figure shows the ratio $r_{t}$ as a function of the Reynolds number for the S and A instabilities (note the log-log scale). The ratio increases steadily with the Reynolds number in both cases, making the frozen baseflow approach all the more legitimate as the Reynolds number is high. 
the frozen baseflow approach questionable. However as soon as the Reynolds number is increased, the variation of $r_{t}$ makes the hypothesis clearly reliable. For instance, the limit $r_{t}>100$ is obtained for $R e>1000$ in the A case and $R e>3000$ in the $\mathrm{S}$ case. The existence of this higher range of Reynolds number for which the frozen baseflow approach is fully legitimate fundamentally supports the importance of the results obtained in the lower range of Reynolds number.

The importance of viscous diffusion in the development of modes S and A has been highlighted. The dependency of the mode on viscosity is supported by the scaling of the wake size on $R e^{-1 / 2}$. Viscous diffusion has been proposed as the mechanism by which vorticity is taken from the displacement modes into the wake. In spite of these results, the viscous nature of the present instabilities remains to be fully resolved. In particular, no scaling on the Reynolds number could be found concerning the growth rate of the instabilities. An investigation applied to a more extended range of Reynolds number would be needed to conclude on this point. The present computational method was limited to rather low Reynolds numbers. The question of viscosity also relates to that of the aspect ratio $a / b$ since in the evolution of a real dipole, the aspect ratio and the Reynolds number evolve together. This point has been rapidly looked upon in Appendix A by considering the case of a smooth dipole. The smooth dipoles also exhibits the modes $\mathrm{S}$ and $\mathrm{A}$ showing that these instabilities are not limited to the LCD.

The existence of an optimal Reynolds number in terms of amplification rate can be analysed based on the proposed scenario of viscous leaking at the oval. When the Reynolds number is increased, this leaking is reduced and, owing to the constraint of impulse, the dipole motion becomes more constrained. Moreover, the wake contribution is reduced. These trends favor a reduction of the growth rate and of the frequency as is observed in the results (see Figures 4 and 7) when the Reynolds number is large enough. Inversely, when the Reynolds number is decreased, viscous diffusion dominates all the dynamics and renders any motion impossible. The optimal growth rate likely results from these conflicting effects of viscosity in the small and large Reynolds number regimes.

Another question is that of the 3D effect on the instabilities. Both modes were shown to belong to a family of 3D unstable modes. In fact, as noted by Moore and Saffman, ${ }^{8}$ bending of the vortex has a stabilizing influence, which agrees with the reduction of the growth rate displayed in Figure 7 and Figure 4 with $k$. In 3D, part of the strain is used to bend the vortices, and self-induction is triggered which induces the rotation of the displacement modes and a less favorable orientation about the axis of strain. The self-rotation also explains why the frequency of mode $\mathrm{A}$ increases with $k$, since displacement modes become retrograde at finite $k$.

Owing to the importance of the wake in the present instabilities, we have made an attempt to compare mode A with the stability of the flow past a cylinder. Indeed, the LCD flow has some similarity with the cylinder, even if the cylinder yields a recirculation flow which is the trigger of the instabilities above a critical Reynolds number approximately equal to 50 (see Williamson ${ }^{21}$ ). In spite of this difference, it has been found that the form of the A mode, displayed in Figure 21 with a blanking of the dipole, bears a visual similarity with the unstable mode at $R e=100$ found by Barkley $^{22}$ (see Figure 3(a) in his article). Moreover, one can also note that the normalized frequency of the antisymmetric mode has a value close to 0.2 (exactly $f^{*}=0.22$, see Figure 7(b)), which is the typical value of the vortex shedding behind a cylinder. The comparison can be brought further by considering the case of the cylinder free to move horizontally and vertically, which is closer to the present flow case, since the dipole features these degrees of freedom. Dahl et al. ${ }^{23}$ has investigated this free cylinder case experimentally and theoretically, and he showed in particular that the trajectory

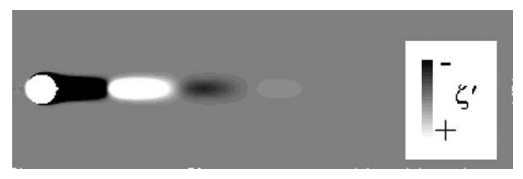

FIG. 21. Wake associated with the $2 \mathrm{D}$ antisymmetric instability. The dipole has been blanked to simulate the presence of a cylinder. 
of the cylinder forms a figure eight pattern, very similar to the result already shown in Figure 8(a). This analogy with the cylinder flow supports the physical scenario about the strong effect of the wake on the dipole dynamics.

\section{ACKNOWLEDGMENTS}

I would like to thank P. Meliga and O. Marquet for their helpful comments on this work, and H. Johnson for her careful re-reading of the paper. Reviewers' comments and discussions have been highly appreciated. Their careful analysis greatly contributed to improve the paper.

\section{APPENDIX A: CASE OF THE SMOOTH DIPOLE}

The case of a smooth dipole has been considered in order to evaluate the possible influence of the sharp vorticity profile of the LCD at the oval boundary upon the stability results in $2 \mathrm{D}$. The baseflow is obtained by a numerical simulation started from an initial state made of two opposite Lamb-Oseen vortices as done by Sipp et al. ${ }^{15}$ The vorticity profile of the smooth dipole along the vertical direction is displayed in Figure 22 and compared to that of the LCD. The smooth dipole, characterized by $a / b=0.3$ is very similar to the LCD profile except at the oval boundary where the vorticity is continuously differentiable.

We performed the same stability analysis as in the LCD case, however only considering $k=0$. Similar 2D unstable modes were found for $R e=223$ in case A and $R e=1280$ in case $\mathrm{S}$. In case A, the Reynolds number is that of the largest growing mode as in Figure 8. In case S, the Reynolds number chosen for Figure 2 is close to the Reynolds number where the maximum growth rate is reached. The axial vorticity field of mode A is shown in Figure 23, and mode S is shown in Figure 24. There is a good agreement compared to Figures 5 and 8 . Note however that the relative amplitudes of the imaginary part are changed compared to the LCD.

The growth rate $\sigma$ and frequency $f$ are given in Table I in normalized units (see (16) for the definition of the normalized quantities). In the two symmetry cases, the growth rate is lower than for the LCD. Following the physical analysis that it is the wake that is responsible for the instabilities, this reduction is coherent. Indeed, the wake shedding is expected to be much lower in the smooth

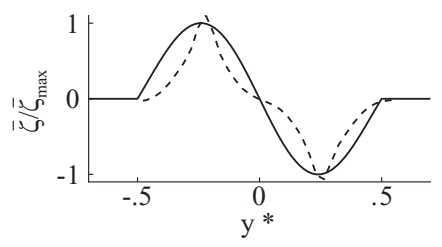

FIG. 22. Comparison of the vorticity profile in the y-direction between the LCD (bold line) and the smooth dipole (dashed line).
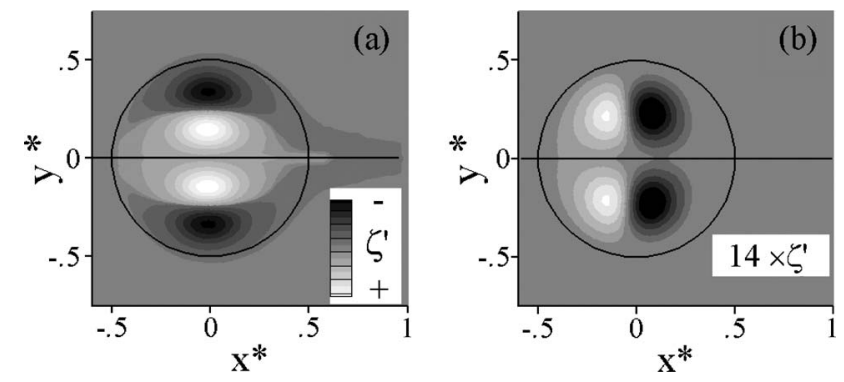

FIG. 23. Mode A calculated for the smooth base flow $(a / b=0.3)$. (a) Real part of mode A. (b) Imaginary part of mode A. The iso-contours in frame (b) have been increased 14 times to use the same iso-levels as in frame (a). 

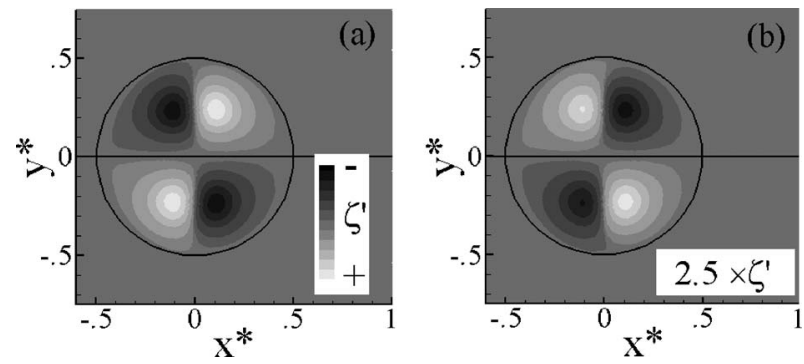

FIG. 24. Mode $\mathrm{S}$ calculated for the smooth base flow $(a / b=0.3)$. (a) Real part of mode S. (d) Imaginary part of mode $\mathrm{S}$. The iso-contours in frame (b) have been increased 2.5 times to use the same iso-levels as in frame (a).

TABLE I. Normalized growth rate and frequency of the $\mathrm{S}$ and A modes of the smooth dipole at $k=0$ compared to the values obtained for the LCD. The values are normalized on the values of $U$ and $D$ associated with the smooth dipole as in (16).

\begin{tabular}{|c|c|c|c|c|}
\hline & \multicolumn{2}{|c|}{$\begin{array}{c}\text { Mode S } \\
R e=1280\end{array}$} & \multicolumn{2}{|c|}{$\begin{array}{c}\text { Mode A } \\
R e=223\end{array}$} \\
\hline & Smooth dipole & LCD & Smooth dipole & LCD \\
\hline$\sigma^{*}$ & 0.025 & 0.042 & 0.400 & 0.690 \\
\hline$f^{*}$ & 0.020 & 0.000 & 0.080 & 0.200 \\
\hline
\end{tabular}

dipole case since the displacement mode exhibits lower vorticity levels close to the oval boundary (it is more concentrated due to the less extended baseflow vorticity).

Concerning the frequency, we note that for mode A it is significantly reduced. This again can be attributed to the weaker vortex shedding and the consequently reduced wake contribution. In case $\mathrm{S}$, the instability becomes unsteady, meaning that there is a slow modulation between the real and the imaginary parts. The triggering of an unsteady S mode likely results from a dissymmetry in the viscous dissipation of the displacement modes, which was not present in the case of the LCD.

\section{APPENDIX B: NUMERICAL VALIDATION}

The numerical method has been validated against the results obtained by Billant et al. ${ }^{2}$ Figure 25 shows the spectra obtained by our method superposed to those obtained by Billant ${ }^{2}$ at Reynolds number $R e=800$. In Billant's ${ }^{2}$ article, the Reynolds number is defined by $R e_{\text {Billant }}=U R / v$ where $R=D / 2$. As a consequence, the present Reynolds number $R e=800$ corresponds to Billant's ${ }^{2}$ Reynolds number equal to 400 . The agreement is very satisfactory. All the modes found by Billant ${ }^{2}$ are recovered by our method.
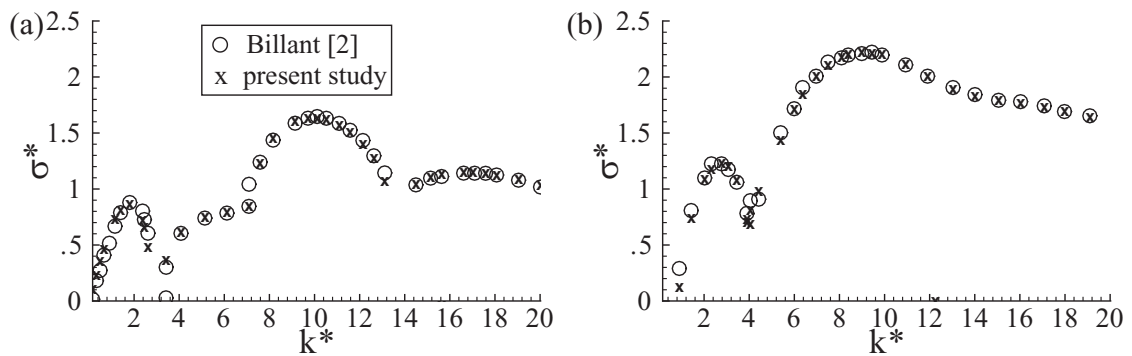

FIG. 25. Validation of the three-dimensional spectra against the results of Billant et al. ${ }^{2}$ (a) Symmetric and (b) antisymmetric spectra at Reynolds 800 which corresponds to the Reynolds number used by Billant ${ }^{2}$ equal to 400 . 
${ }^{1}$ V. Meleshko and G. van Heijst, "On Chaplygin's investigations of two-dimensional vortex structures in an inviscid fluid," J. Fluid Mech. 272, 157-182 (1994).

${ }^{2}$ P. Billant, P. Brancher, and J. Chomaz, "Three-dimensional stability of a vortex pair," Phys. Fluids 11, 2069 (1999).

${ }^{3}$ P. Saffman, Vortex Dynamics (Cambridge University Press, Cambridge, UK, 1992).

${ }^{4}$ P. Saffman and R. Szeto, "Equilibrium shapes of a pair of equal uniform vortices," Phys. Fluids 23, 2339-2342 (1980).

${ }_{6}^{5}$ S. Crow, "Stability theory for a pair of trailing vortices," AIAA J. 8, 2172-2179 (1970).

${ }^{6}$ J. van Geffen and G. van Heijst, "Viscous evolution of 2D dipolar vortices," Fluid Dyn. Res. 22, 191-213 (1998).

${ }^{7}$ I. Delbende and M. Rossi, “The dynamics of a viscous vortex dipole," Phys. Fluids 21, 073605 (2009).

${ }^{8}$ D. Moore and P. Saffman, "Structure of a line vortex in an imposed strain," Aircraft Wake Turbulence and its Detection (Springer, New York, 1971), pp. 339-354.

${ }^{9}$ A. Robinson and P. Saffman, "Three-dimensional stability of an elliptical vortex in a straining field," J. Fluid Mech. 142, 451-466 (1984).

${ }^{10}$ M. Wells and G. van Heijst, "A model of tidal flushing of an estuary by dipole formation," Dyn. Atmos. Oceans 37, 223-244 (2003).

${ }^{11}$ W. de Ruijter, H. Aken, E. Beier, J. Lutjeharms, R. Matano, and M. Schouten, "Eddies and dipoles around South Madagascar: Formation, pathways and large-scale impact," Deep-Sea Res. Part I 51, 383-400 (2004).

${ }^{12}$ R. Khvoles, D. Berson, and Z. Kizner, "The structure and evolution of elliptical barotropic modons," J. Fluid Mech. 530, $1-30$ (2005).

${ }^{13}$ D. Dritschel, "A general theory for two-dimensional vortex interactions," J. Fluid Mech. 293, 269-303 (1995).

${ }^{14}$ J. Hesthaven, J. Lynov, A. Nielsen, J. Rasmussen, M. Schmidt, E. Shapiro, and S. Turitsyn, "Dynamics of a nonlinear dipole vortex," Phys. Fluids 7, 2220 (1995).

${ }^{15}$ D. Sipp, L. Jacquin, and C. Cosssu, "Self-adaptation and viscous selection in concentrated two-dimensional vortex dipoles," Phys. Fluids 12, 245 (2000).

${ }^{16}$ F. Hecht, "New development in freefem++," J. Numer. Math. 20, 251-265 (2012).

${ }^{17} \mathrm{C}$. Tsai and S. Widnall, "The stability of short waves on a straight vortex filament in a weak externally imposed strain field," J. Fluid Mech. 73, 721-733 (1976).

${ }^{18}$ D. Sipp and L. Jacquin, "Widnall instabilities in vortex pairs," Phys. Fluids 15, 1861-1874 (2003).

${ }^{19}$ D. Fabre, D. Sipp, and L. Jacquin, "Kelvin waves and the singular modes of the Lamb-Oseen vortex," J. Fluid Mech. 551, 235-274 (2006).

${ }^{20}$ S. Widnall, D. Bliss, and A. Zalay, "Theoretical and experimental study of the stability of a vortex pair," Aircraft Wake Turbulence and its Detection (Springer, New York, 1971), pp. 305-338.

${ }^{21}$ C. Williamson, "Vortex dynamics in the cylinder wake," Annu. Rev. Fluid Mech. 28, 477-539 (1996).

${ }^{22}$ D. Barkley, "Linear analysis of the cylinder wake mean flow," EPL 75, 750 (2006).

${ }^{23}$ J. Dahl, F. Hover, M. Triantafyllou, S. Dong, and G. Karniadakis, "Resonant vibrations of bluff bodies cause multivortex shedding and high frequency forces," Phys. Rev. Lett. 99, 144503 (2007). 